\title{
Inflammasome activation: from inflammatory disease to infection
}

\author{
Aisling Dunne ${ }^{1}$ \\ School of Biochemistry and Immunology, and School of Medicine, Trinity College Dublin, Dublin 2, Ireland
}

\begin{abstract}
The recognition of pathogen-derived molecules by the innate immune system is mediated by a number of receptors, including members of the TLR (Toll-like receptor), RLH [RIG (retinoic acid-inducible gene)-like helicase] and the NLR (NOD-like receptor) families. NLRs in particular are also involved in the recognition of host-derived 'danger'-associated molecules which are produced under conditions of cellular stress or injury. Activation of these receptors leads to the assembly of high-molecular-mass complexes called inflammasomes which in turn leads to the generation of active caspase 1 and to the production of mature IL$1 \beta$ (interleukin $1 \beta$ ). The discovery that NLRP3 (NLR-related protein 3) can recognize host-derived particulate matter such as uric acid and cholesterol crystals has led to this inflammasome being implicated in a number of inflammatory diseases, including gout, atherosclerosis and Type 2 diabetes. In addition, aberrant NLRP3 activation has also been observed in a number of heritable disorders now referred to as cryopyrinopathies. On the other hand, a number of studies have reported that recognition of both viral and bacterial products by NLRs is required for effective pathogen clearance. The present review discusses both aspects of NLR activation and will highlight the role of additional inflammasome complexes in sensing infection.
\end{abstract}

\section{Introduction}

Originally termed endogenous or leucocyte pyrogen, on the basis of its ability to induce fever, the role of IL (interleukin)$1 \beta$ in driving pro-inflammatory reactions has long been recognized [1]. Over the last 10 years, however, there has been a resurgence of interest in this cytokine following the discovery of the molecular platform which regulates the maturation of not only this protein, but also IL-18. Both cytokines lack a signal sequence and are first produced as inactive precursors which must be cleaved into a mature and active form before being secreted from the cell. This cleavage event is catalysed by caspase 1 and, in the case of IL- $1 \beta$, a deregulation of this process results in a number of autoinflammatory syndromes, examples of which include FCAS (familial cold autoinflammatory syndrome), MWS (Muckle-Wells syndrome) and CINCA (chronic infantile cutaneous neurological articular syndrome) [2]. Indeed, one of the defining features of autoinflammatory disease is that blockade of IL-1 rather than TNF $\alpha$ (tumour necrosis factor $\alpha)$ usually results in a rapid cessation of symptoms, hence administration of the IL-1 receptor antagonist Anakinra or blockade of the IL-1 receptor itself represents an effective treatment for many of these disorders. In addition to

Key words: infection, inflammasome, inflammatory disease, innate immunity, interleukin $1 \beta$ (IL-1 $\beta)$, NOD-like receptor.

Abbreviations used: AIM2, absent in melanoma 2; ASC, apoptosis-associated speck-like protein containing a caspase-recruitment domain; CARD, caspase recruitment domain; FCAS, familia cold autoinflammatory syndrome; IAPP, islet amyloid precursor protein; IL, interleukin; IPAF, IL-1 $\beta$-converting enzyme protease-activating factor; IRF, interferon regulatory factor; LPS lipopolysaccharide; mCMV, mouse cytomegalovirus; MWS, Muckle-Wells syndrome; NLR, NODlike receptor; NLRP, NLR-related protein; RIG, retinoic acid-inducible gene; ROS, reactive oxygen species; TLR, Toll-like receptor

'email aidunne@tcd.ie autoinflammatory disease, IL-1 plays a central role in combating infection and has also been implicated in the pathogenesis of autoimmune disease, where it is has been shown to promote the induction or expansion of pathogenic Th17 cells [3]. The present review focuses on the events involved in the generation of active IL- $1 \beta$, a tightly regulated process that governs the outcome of not only inflammatory disease, but also bacterial, viral and fungal infection.

\section{IL-1 $\beta$ and autoinflammatory disease}

A key finding linking excessive IL-1 production to autoinflammatory disease came with the identification of a gain-of-function mutation in a novel pyrin-like protein in patients presenting with FCAS and MWS [4]. The protein, later named NLRP3 [NLR (NOD-like receptor)-related protein 3], belongs to a larger family of cytosolic receptors termed NLRs and was subsequently shown to form part of a high-molecular-mass IL- $1 \beta$-processing complex called the inflammasome [5]. Caspase 1, the adapter protein ASC [apoptosis-associated speck-like protein containing a CARD (caspase-recruitment domain)] and the CARD-containing protein Cardinal were also identified as components of this complex, which was subsequently shown to be activated by ATP, bacterial pore-forming toxins and gout-associated uric acid and calcium pyrophosphate crystals [6,7]. Hence, it soon became apparent that NLRP3 was not recognizing a single ligand, but rather was being activated in response to an insult or injury to the target cell. Since these early studies, a great deal of progress has been made. To date, 22 NLRs have been identified in humans, and 34 have been identified in mice [8]. All members of the family contain C-terminal leucine-rich 
Figure 1 | Schematic representation of inflammasome complexes

Inflammasome activation leads to the generation of active caspase 1 , which in turn cleaves the pro-form of IL-1 $\beta$ into a mature and active form. Unrestrained ligand-independent inflammasome activation or recognition of host-derived ligands leads to autoinflammatory and inflammatory disease respectively, whereas recognition of pathogen-derived molecules is required for infection clearance. FIIND, domain with a function to find; HIN200, haemopoietic interferon-inducible nuclear antigens with 200 amino acid repeats; LRR, leucine-rich repeat; MDP, muramyl dipeptide; NALP, NACHT, leucine-rich repeat and pyrin domain-containing protein.

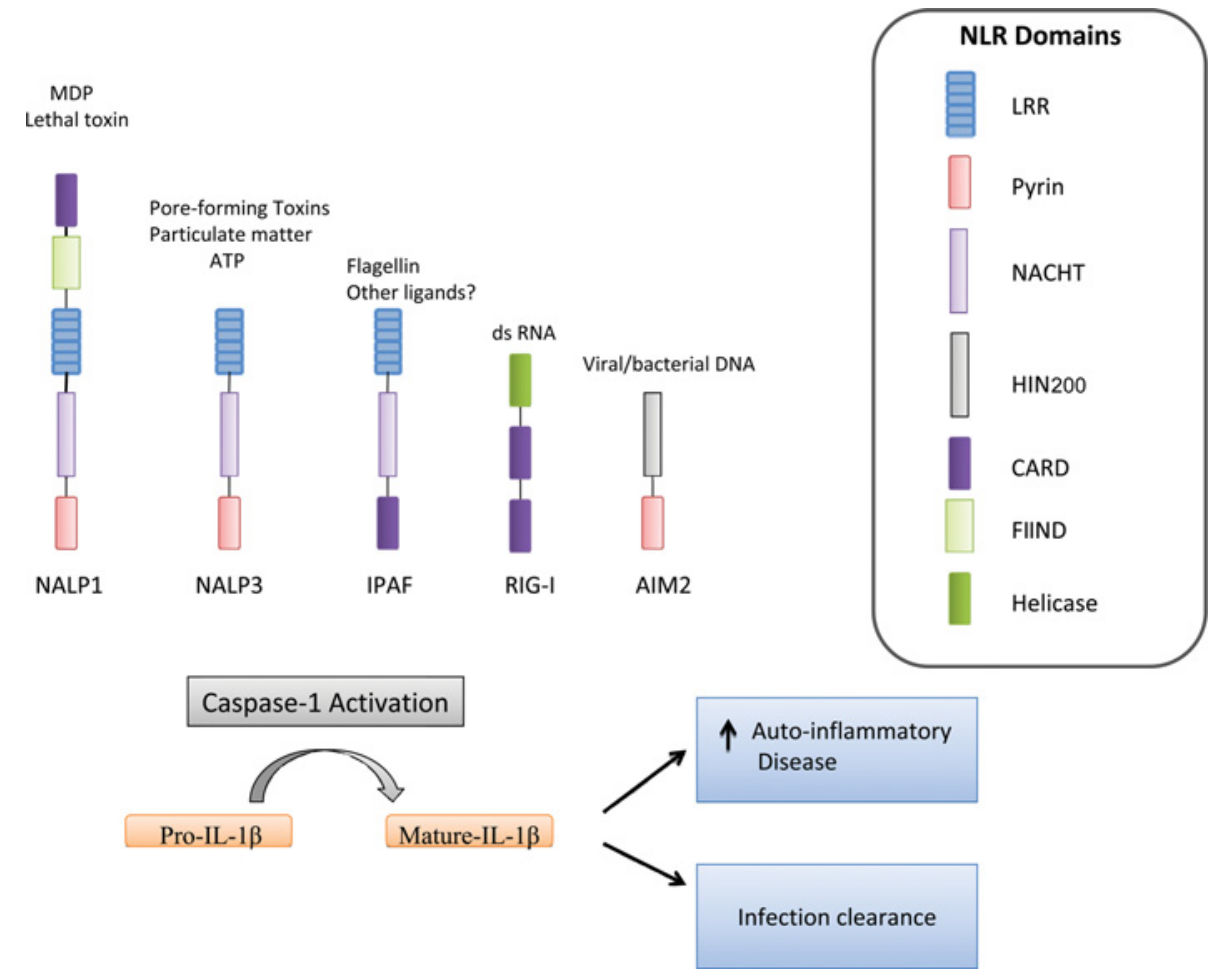

repeats followed by a nucleotide-binding NACHT domain, but they differ in their N-terminal effector domain (Figure 1). NLRP3 contains a pyrin-like domain which is required for the recruitment of the adaptor molecule ASC which also harbours this moiety.

In addition to NLRP3, NLRP1, RIG-1 (retinoic acidinducible gene 1), AIM2 (absent in melanoma 2) and IPAF (IL-1 $\beta$-converting enzyme protease-activating factor) inflammasomes have also been identified; however, NLRP3 remains the most extensively characterized. The precise mechanism of activation has yet to be determined; however, there are common events involved. For example, all of the known inflammasome activators induce a reduction in intracellular potassium levels hence pre-treatment of cells with $\mathrm{K}^{+}$channel blockers such as quinidine prevents IL$1 \beta$ processing. Inhibitors of ROS (reactive oxygen species) generation also block inflammasome activation; however, macrophages lacking phagosomal NADPH oxidase respond normally to NLRP3 agonists, suggesting a possible functional redundancy between NADPH oxidase family members or the existence of alternative sources of ROS generation. Indeed, it was recently reported that the inflammasome is positively regulated by ROS generated by mitochondrial damage [9], thus providing a plausible link between mitochondrial dysfunction and inflammatory disease. In the case of particulate matter such as uric acid crystals, it is believed that, following phagocytosis, lysosomes are unable to process the insoluble material, which results in lysosomal rupture and the release of proteases such as cathepsins, which then go on to cleave an endogenous NLRP3 ligand [10]. It is possible also that these proteases are cleaving an inhibitory domain of NLRP3 itself, thus releasing the protein from an inactive conformation. Indeed, the list of particulate matter capable of activating the inflammasome is steadily growing and now includes silica crystals, cholesterol crystals, which are associated with atherosclerosis, $\beta$-amyloid, which is associated with Alzheimer's disease, and IAPP (islet amyloid precursor protein), which is linked to Type 2 diabetes.

In addition to inflammasome activation, a priming signal is also required to drive the production of active IL-1. This signal serves to drive the transcription of the pro-form of IL$1 \beta$ and can be mediated by a TLR (Toll-like receptor) agonist such as LPS (lipopolysaccharide), which is commonly used in experimental settings. The nature of this signal in an in vivo context is the subject of ongoing investigation, as there is little evidence linking infection to autoinflammatory disease. In the 
case of uric acid crystals, it has recently been demonstrated that unbound fatty acids can engage TLR2 and synergize with the crystals to drive the production of active IL-1, thus providing a link between copious food consumption and acute attacks of gout [11]. However, it should be noted that, in this study, joint inflammation following injection of uric acid crystals was found to be NLRP3-independent. We have also recently reported that glucose metabolism and minimally oxidized low-density lipoprotein, which is more abundant in diabetic dyslipidaemia, can act as a primer for IAPP-induced inflammasome activation [12]. It remains to be seen whether low levels of LPS derived from commensals can act as priming agents in autoinflammatory disease where NLRP3 is active in the absence of a ligand.

\section{Inflammasome activation and infection}

As well as endogenously derived 'danger' molecules, NLRP3 is also activated by microbial products. Nigericin, a microbial toxin derived from Streptomyces bygroscopicus, and the marine toxin maitotoxin were the first such molecules described [6]. We have also recently demonstrated that adenylate cyclase toxin (CyaA), a key virulence factor from Bordetella pertussis, activates the NLRP3 inflammasome and that CyaA-driven IL- $1 \beta$ production is required for the generation of antigen-specific IL-17 [13]. This in agreement with studies in mice where targeted mutations in NLRP3 (NACHT, leucine-rich repeat and pyrin domain-containing protein 3), leading to inflammasome hyperactivation, similar to that seen in MWS, not only resulted in excessive IL-1 $\beta$ production, but also enhanced Th17 cells [14]. Thus innate inflammatory responses activated through the inflammasome, considered to be pathogenic in many disease settings, may also promote protective adaptive immunity to pathogens.

It has recently been demonstrated that NLRP3 is required for protective immunity against Streptococcus pneumoniae respiratory infection, and the virulence factor pneumolysin, which also exhibits pore-forming activity, has been identified, in this case as an NLRP3 activator [15]. NLRP3 has also been shown to play a central role in regulating caspase 1 activation in response to Listeria monocytogenes [16] and chlamydial infection [17]. Indeed, manipulation of inflammasome activation during bacterial infection has also been demonstrated. For example, infection of human monocytes with the intracellular bacterium Legionella pneumophila leads to a down-regulation of the adapter molecule ASC and a reduced capacity to generate protective IL-I $\beta$, thus leading to prolonged survival of the bacterium [18]. A number of T3SS (type III secretion system) effector molecules such as YopE and YopT from Yersinia enterocolitica have also been reported to inhibit inflammasome activation and, in some cases, these effects have been attributed to the ability of these molecules to modulate caspase 1 oligomerization (reviewed in detail in [19]).

In addition to bacterial infection, NLRP3 has also been reported to play a crucial role in host defence against the fungal pathogen Candida albicans [20,21]. In this instance, TLR2 and Dectin-1 are required to drive the transcription of the pro-form of IL- $1 \beta$, thus providing the priming signal (signal 1), whereas NLRP3 was required for caspase-1induced processing of the immature cytokine. Furthermore, genetic deletion or pharmacological inhibition of the nonreceptor tyrosine kinase Syk, which operates downstream of ITAM (immunoreceptor tyrosine-based activation motif)coupled fungal pattern recognition receptors selectively abrogated inflammasome activation by C. albicans [21], thus implicating C-type lectin receptors in inflammasomemediated host defence. Similarly, the helminth parasite Schistoma mansoni was found to trigger Dectin-2 and NLRP3 inflammasome activation in a Syk-dependent manner, and, as is the case for Candida infection, this activity is dependent on ROS generation and $\mathrm{K}^{+}$efflux [22].

NLRP3 has also been implicated in the immune response to viral infection (reviewed in detail in [23]). For example, in an animal model of influenza infection, NLRP3-deficient mice were found to have increased mortality and defective neutrophil influx into the lung compared with wild-type mice $[24,25]$. Although it was demonstrated previously that administration of purified influenza $A$ virus RNA was sufficient to drive NLRP3-dependent IL- $1 \beta$ secretion and inflammation, a subsequent study demonstrated that viral RNA alone was insufficient to trigger inflammasome activation and that a Golgi-localized virus-encoded ion channel (M2) was required for this effect [26]. It has been suggested that a deregulated ionic balance in the Golgi leads to the activation of plasma membrane ion channels, resulting in $\mathrm{K}^{+}$efflux from target cells and activation of NLRP3 in a manner similar to bacterial pore-forming toxins. The priming signal was shown to be mediated by the endosomal RNA receptor TLR7, which is capable of activating NF- $\kappa \mathrm{B}$ (nuclear factor $\kappa \mathrm{B}$ ) and hence driving the production of pro-IL- $1 \beta$.

Inhibition of inflammasome activation by influenza A virus has also been demonstrated. In this case, infection of primary macrophages with virus encoding a mutant form of NS1, which is known to suppress host antiviral responses by multiple effector mechanisms, resulted in the release of high levels of IL-1 $\beta$ and IL-18 [27]. NLRP3 was also found to mediate innate immune responses to MVA (modified vaccina virus Ankara), in conjunction with the patternrecognition receptors TLR2 and TLR6 and the RNA sensor MDA-5 (melanoma differentiation-associated gene 5) [28]. An early study performed before the identification of the inflammasome demonstrated that the vaccina virus protein B13R could inhibit the processing of IL-1 $\beta$ [29] in infected monocytes, thus it is likely that multiple viruses have evolved mechanisms to inhibit inflammasome-mediated cytokine production. Indeed, inhibition of the inflammasome is not exclusive to pathogens, and the host itself has also evolved mechanisms to block activation once the appropriate immune response has been mounted. The cytosolic protein pyrin interacts with NLPR 3 and caspase 1 and, in so doing, attenuates IL-1 $\beta$ processing. In accordance with this, individuals bearing mutations in $M E F V$, the gene encoding pyrin, suffer from 
FMF (familial Mediterranean fever), which is characterized by fever, abdominal or chest pain and arthritis [2].

\section{Alternative inflammasome complexes}

The cytosolic receptors RIG-1 and AIM2 have also been shown to assemble into caspase-1-activating inflammasome complexes. RIG-1, a member of the RLH (RIG-like helicase) family, was implicated previously in the generation of type I interferons in response to viral RNA via activation of the transcription factors IRF (interferon regulatory factor) 3 and IRF7 [30]; however, it has been demonstrated more recently that RIG-1 can also induce the production of active IL- $1 \beta$ and IL-18 in a caspase-1-dependent manner [31]. AIM2, a member of the HIN200 family of proteins, has been identified as a sensor of both bacterial and viral dsDNA (doublestranded DNA) [32-34]. Mice deficient for this receptor were unable to mount effective responses to the intracellular bacterium Francisella tularensis [33,34]. Similarly AIM2deficient mice have defective responses to the DNA viruses, vaccinia virus and mCMV (mouse cytomegalovirus) [34]. A role for AIM2 in driving IL-18 and natural-killer-celldependent interferon- $\gamma$ production which is required for the early control of mCMV infection was also confirmed in vivo. Finally, it was demonstrated that both AIM2 and NLRP3 are required for L. monocytogenes-mediated inflammasome activation [16]. A two-step model was proposed whereby NLRP3 is activated following phagocytic uptake of the bacterium and release of the phagolysosomal content in a process mediated by the pore-forming cytolysin listeriolysin $\mathrm{O}$. The replicating bacterium then translocates to the cytosol where Listeria-derived DNA is sensed by AIM2.

Additional non-viral sensing inflammasomes include NLRP1 and IPAF. NLRP1 is highly polymorphic, and mice bearing the NLRP1b locus are susceptible to Bacillus anthracis lethal toxin [35]. NLRP1 also responds to the bacterial cell wall component MDP (muramyl dipeptide), together with the NLR family member NOD-2. In humans, polymorphisms in NLRP1 have been associated with a number of autoinflammatory and autoimmune disorders, including latent autoimmune diabetes in adults, rheumatoid arthritis, psoriasis and systemic lupus erythaematosus [36]; however, the basis of disease pathogenesis has yet to be determined. IPAF is activated in response to a number of bacteria possessing type III or type IV secretion systems such as L. pneumophila and Salmonella enterica serotype Typhimurium [37,38]. The bacterial protein flagellin, which is translocated into the cytosolic by these secretion systems, is sensed by IPAF, although there is also evidence to suggest that IPAF is activated by additional ligands, since nonflagellated bacteria such as Shigella flexneri can activate the this inflammasome [39].

\section{Concluding remarks}

The inflammasome is clearly a central player in perpetuating the host innate immune response to both infectious and non-infectious matter. A number of questions remain surrounding the events that occur upstream of NLRP activation. Although ionic imbalance, ROS generation and lysosomal rupture are all known to generate an active IL-1processing complex, the molecular consequences in terms of complex assembly are still unclear. It is likely that a common second messenger or effector molecule is being generated or activated, given the ability of NLRPs to respond to such a diverse range of stimuli. As mentioned above, the nature of the priming signal in so-called 'sterile' inflammation is also of particular interest and, as has been highlighted in recent studies, metabolic products are likely candidates. In addition, a number of NLR family members remain uncharacterized, therefore the identification of disease associations may be of benefit in determining their function. The discovery that excessive inflammasome activation contributes to the pathogenesis of a number of autoinflammatory diseases has also prompted the development of next-generation IL-1 inhibitors, including antibodies that specifically neutralize IL- $1 \beta$, leaving IL- $1 \alpha$ intact to signal and mediate responses to infection. Future efforts will no doubt lead to the generation of small-molecule inhibitors that target inflammasome components directly and block not only IL- $1 \beta$, but also IL18 , which is required for the generation of adaptive immune responses.

\section{Funding}

Supported by Science Foundation Ireland and the Immunology Research Centre Trinity College Dublin.

\section{References}

1 Dinarello, C.A. (2009) Immunological and inflammatory functions of the interleukin-1 family. Annu. Rev. Immunol. 27, 519-550

2 Masters, S.L., Simon, A., Aksentijevich, I. and Kastner, D.L. (2009) Horror autoinflammaticus: the molecular pathophysiology of autoinflammatory disease. Annu. Rev. Immunol. 27, 621-668

3 Sutton, C., Brereton, C., Keogh, B., Mills, K.H. and Lavelle, E.C. (2006) A crucial role for interleukin (IL)-1 in the induction of IL-17-producing $T$ cells that mediate autoimmune encephalomyelitis. J. Exp. Med. 203 1685-1691

4 Hoffman, H.M., Mueller, J.L., Broide, D.H., Wanderer, A.A. and Kolodner, R.D. (2001) Mutation of a new gene encoding a putative pyrin-like protein causes familial cold autoinflammatory syndrome and Muckle-Wells syndrome. Nat. Genet. 29, 301-305

5 Agostini, L., Martinon, F., Burns, K., McDermott, M.F., Hawkins, P.N. and Tschopp, J. (2004) NALP3 forms an IL-1 $\beta$-processing inflammasome with increased activity in Muckle-Wells autoinflammatory disorder. Immunity 20, 319-325

6 Mariathasan, S., Weiss, D.S., Newton, K., McBride, J., O'Rourke, K., Roose-Girma, M., Lee, W.P., Weinrauch, Y., Monack, D.M. and Dixit, V.M. (2006) Cryopyrin (NALP3) activates the inflammasome in response to toxins and ATP. Nature $\mathbf{4 4 0}, 228-232$

7 Martinon, F., Pétrilli, V., Mayo, A., Tardive, A. and Tschopp, J. (2006) Gout-associated uric acid crystals activate the NALP3 inflammasome. Nature 440, 237-241

8 Shaw, P.J., Lamkanf, M. and Kanneganti, T.D. (2010) NOD-like receptor (NLR) signaling beyond the inflammasome. Eur. J. immunol. 40, 595-653

9 Zhou, R., Yazdi, A.S., Menu, P. and Tschopp, J. (2011) A role for mitochondria in NLRP3 inflammasome activation. Nature 469, 221-225

10 Jin, C. and Flavell, R.A. (2010) Molecular mechanism of in NLRP3 inflammasome activation. J. Clin. Immunol. 30, 628-631 
11 Joosten, L.A., Netea, M.G., Mylona, E., Koenders, M.I., Malireddi, R.K., Oosting, M., Stienstra, R., van de Veerdonk, F.L., Stalenhoef, A.F., Giamarellos-Bourboulis, E.J. et al. (2010) Engagement of fatty acids with Toll-like receptor 2 drives interleukin- $1 \beta$ production via the ASC/caspase 1 pathway in monosodium urate monohydrate crystal-induced gouty arthritis. Arthritis Rheum. 62, 3237-3248

12 Masters, S.L., Dunne, A., Subramanian, S.L., Hull, R.L., Tannahill, G.M., Sharp, F.A., Becker, C., Franchi, L., Yoshihara, E., Chen, Z. et al. (2010) Activation of the NLRP3 inflammasome by islet amyloid polypeptide provides a mechanism for enhanced IL-1 $\beta$ in type 2 diabetes. Nat. Immunol. 11, 897-904

13 Dunne, A., Ross, P.J., Pospisilova, E., Masin, J., Meaney, A., Sutton, C. Tschopp, J., Sebo, P. and Mills, K.H. (2010) IL-1 $\beta$ induction via toxin-mediated activation of the inflammasome promotes Th17 responses and protective immunity to Bordetella pertussis. J. Immunol 185, 1711-1719

14 Meng, G., Zhang, F., Fuss, I., Kitani, A. and Strober, W. (2009) A mutation in the N/rp3 gene causing inflammasome hyperactivation potentiates Th17 cell-dominant immune responses. Immunity 30, 860-874

15 McNeela, E.A., Burke, A., Neill, D.R., Baxter, C., Fernandes, V.E., Ferreira, D., Smeaton, S., El-Rachkidy, R., McLoughlin, R.M., Mori, A. et al. (2010) Pneumolysin activates the NLRP3 inflammasome and promotes proinflammatory cytokines independently of TLR4. PLoS Pathog. 6 e1001191

16 Kim, S., Bauernfeind, F., Ablasser, A., Hartmann, G., Fitzgerald, K.A. Latz, E. and Hornung, V. (2010) Listerio monocytogenes is sensed by the NLRP3 and AIM2 inflammasome. Eur. J. Immunol. 40, 1545-1551

17 Abdul-Sater, A.A., Saïd-Sadier, N., Padilla, E.V. and Ojcius, D.M. (2010) Chlamydial infection of monocytes stimulates IL-1 $\beta$ secretion through activation of the NLRP3 inflammasome. Microbes Infect. 12, 652-661

18 Abdelaziz, D.H., Gavrilin, M.A., Akhter, A., Caution, K., Kotrange, S., Khweek, A.A., Abdulrahman, B.A., Grandhi, J., Hassan, Z.A., Marsh, C. et al. (2011) ASC controls Legionello pneumophilo infection in human monocytes. J. Biol. Chem. 286, 3203-3208

19 Taxman, D.J., Huang, M.T.H. and Ting, J.P. (2010) Inflammasome inhibition asa pathogenic stealth mechanism. Cell Host Microbe $\mathbf{8}$, 7-11

20 Gross, 0., Poeck, H., Bscheider, M., Dostert, C., Hannesschläger, N., Endres, S., Hartmann, G., Tardivel, A., Schweighoffer, E., Tybulewicz, V. et al. (2009) Syk kinase signalling couples to the Nlrp3 inflammasome for anti-fungal host defence. Nature 459, 433-436

21 Hise, A.G., Tomalka, J., Ganesan, S., Patel, K., Hall, B.A., Brown, G.D. and Fitzgerald, K.A. (2009) An essential role for the NLRP3 inflammasome in host defense against the human fungal pathogen Condida albicans. Cell Host Microbe 5, 487-497

22 Ritter, M., Gross, 0., Kays, S., Ruland, J., Nimmerjahn, F., Saijo, S., Tschopp, J., Layland, L.E. and Prazeres da Costa, C. (2010) Schistosomo mansoni triggers Dectin-2, which activates the Nlrp3 inflammasome and alters adaptive immune responses. Proc. Natl. Acad. Sci. U.S.A. 107, 20459-20464

23 Kanneganti, T. (2010) Central roles of NLRs and inflammasomes in viral infection. Nat. Rev. Immunol. 10, 688-698

24 Allen, I.C., Scull, M.A., Moore, C.B., Holl, E.K., McElvania-TeKippe, E., Taxman, D.J., Guthrie, E.H., Pickles, R.J. and Ting, J.P. (2009) The NLRP3 inflammasome mediates in vivo innate immunity to influenza A virus through recognition of viral RNA. Immunity 30, 556-565

25 Thomas, P.G., Dash, P., Aldridge, Jr, J.R., Ellebedy, A.H., Reynolds, C., Funk, A.J., Martin, W.J., Lamkanfi, M., Webby, R.J., Boyd, K.L. et al. (2009) The intracellular sensor NLRP3 mediates key innate and healing responses to influenza A virus via the regulation of caspase-1. Immunity $\mathbf{3 0}$, 566-575
26 Ichinohe, T., Pang, I.K. and Iwasaki, A. (2010) Influenza virus activates inflammasomes via its intracellular M2 ion channel. Nat. Immunol. 11, 404-410

27 Stasakova, J., Ferko, B., Kittel, C., Sereinig, S., Romanova, J., Katinger, H. and Egorov, A. (2005) Influenza A mutant viruses with altered NS1 protein function provoke caspase- 1 activation in primary human macrophages, resulting in fast apoptosis and release of high levels of interleukins $1 \beta$ and 18. J. Gen. Virol. 86, 185-195

28 Delaloye, J., Roger, T., Steiner-Tardivel, Q.G., Le Roy, D., Knaup Reymond, M., Akira, S., Petrilli, V., Gomez, C.E., Perdiguero, B., Tschopp, J. et al. (2009) Innate immune sensing of modified vaccinia virus Ankara (MVA) is mediated by TLR2-TLR6, MDA-5 and the NALP3 inflammasome. PLOS Pathog. 5, e1000480

29 Kettle, S., Alcamí, A., Khanna, A., Ehret, R., Jassoy, C. and Smith, G.L. (1997) Vaccinia virus serpin B13R (SPI-2) inhibits interleukin- $1 \beta$-converting enzyme and protects virus-infected cells from TNF- and Fas-mediated apoptosis, but does not prevent IL-1 $\beta$-induced fever. J. Gen. Virol. 78, 677-685

30 Rehwinkel, J., Tan, C.P., Goubau, D., Schulz, O., Pichlmair, A., Bier, K., Robb, N., Vreede, F., Barclay, W., Fodor, E. and Reis e Sousa, C. (2010) RIG-I detects viral genomic RNA during negative-strand RNA virus infection. Cell 140, 397-408

31 Poeck, H., Bscheide, M., Gross, 0., Finge, K., Roth, S., Rebsamen, M., Hannesschläger, N., Schlee, M., Rothenfusser, S., Barchet, W. et al. (2010) Recognition of RNA virus by RIG-I results in activation of CARD9 and inflammasome signaling for interleukin $1 \beta$ production. Nat. Immunol. 11, 63-69

32 Bürckstümmer, T., Baumann, C., Blüml, S., Dixit, E., Dürnberger, G., Jahn, H., Planyavsky, M., Bilban, M., Colinge, J., Bennett, K.L. and Superti-Furga, G. (2009) An orthogonal proteomic-genomic screen identifies AIM2 as a cytoplasmic DNA sensor for the inflammasome. Nat. Immunol. 10, 266-272

33 Fernandes-Alnemri, T., Yu, J.W., Datta, P., Wu, J. and Alnemri, E.S. (2009) AIM2 activates the inflammasome and cell death in response to cytoplasmic DNA. Nature 458, 509-513

34 Hornung, V., Ablasser, A., Charrel-Dennis, M., Bavernfeind, F., Horvath, G., Caffrey, D.R., Latz, E. and Fitzgerald, K.A. (2009) AIM2 recognizes cytosolic dsDNA and forms a caspase-1-activating inflammasome with ASC. Nature 458, 514-518

35 Hsu, L.C., Ali, S.R., McGillivray, S., Tseng, P.H., Mariathasan, S., Humke, E.W., Eckmann, L., Powell, J.J., Nizet, V., Dixit, V.M. and Karin, M. (2008) A NOD2-NALP1 complex mediates caspase-1-dependent IL-1 $\beta$ secretion in response to Bacillus anthracis infection and muramyl dipeptide. Proc. Natl. Acad. Sci. U.S.A. 105, 7803-7808

36 Jin, Y., Mailloux, C.M., Gowan, K., Riccard, S.L., LaBerge, G., Bennett, D.C., Fain, P.R. and Spritz, R.A. (2007) NALP1 in vitiligo-associated multiple autoimmune disease. N. Engl. J. Med. 356, 1216-1225

37 Amer, A., Franchi, L., Kanneganti, T.D., Body-Malapel, M., Ozören, N., Brady, G., Meshinchi, S., Jagirdar, R., Gewirtz, A., Akira, S and Núñez, G. (2006) Regulation of Legionella phagosome maturation and infection through flagellin and host Ipaf. J. Biol. Chem. 281, 35217-35223

38 Franchi, L., Amer, A., Body-Malapel, M., Kanneganti, T.D., Ozören, N., Jagirdar, R., Inohara, N., Vandenabeele, P., Bertin, J., Coyle, A. et al. (2006) Cytosolic flagellin requires Ipaf for activation of caspase-1 and interleukin $1 \beta$ in Solmonello-infected macrophages. Nat. Immunol. 7 576-582

39 Suzuki, T., Franchi, L., Toma, C., Ashida, H., Ogawa, M., Yoshikawa, Y., Mimuro, H., Inohara, N., Sasakawa, C. and Nuñez, G. (2007) Differential regulation of caspase- 1 activation, pyroptosis, and autophagy via Ipaf and ASC in Shigello-infected macrophages. PLoS Pathog. 3, e111

Received 20 December 2010

doi:10.1042/BST0390669 